\title{
COMPACTNESS IN WIENER AMALGAMS ON LOCALLY COMPACT GROUPS
}

\author{
S. S. PANDEY
}

Received 18 December 2002

\begin{abstract}
We study the compactness of bounded subsets in a Wiener amalgam whose local and global components are solid Banach function (BF) spaces on a locally compact group. Our main theorem provides a generalization of the corresponding results of Feichtinger. This paper paves the way for the study of compact multiplier operators on general Wiener amalgams on the lines of Feichtinger.
\end{abstract}

2000 Mathematics Subject Classification: 43A65, 46F05.

1. Introduction. The concept of Wiener amalgams on a locally compact group was introduced by Feichtinger [4] in 1977. In a series of brilliant papers $[4,5,7,8]$, Feichtinger has explored the vital role of Wiener amalgams in general harmonic analysis and its various applications. These spaces, in fact, describe the global and local behaviours of functions or distributions independently and provide very convenient generalizations of the classical function and sequence spaces. Initially, in 1926, Wiener [21] studied a special case of amalgam spaces on the real line in the form of mixed normed spaces. Feichtinger, therefore, in the above-mentioned papers, named them "Wiener-type spaces." But in subsequent papers (cf. [11, 12]) he has used the nomenclature "Wiener Amalgams" and made a distinction from ordinary amalgam spaces (cf. [10, page 395]). Fournier and Stewart [16], in a survey article, have given in detail the construction of ordinary amalgams using $L^{p}$-spaces as local and $L^{q}$-spaces as global components and pointed out a number of applications in classical analysis.

In order to define Wiener amalgams $W(B, Y)\left(R^{m}\right)$ over $m$-dimensional Euclidean spaces, Feichtinger has used the spaces $S\left(R^{m}\right)$ of tempered distributions as a reservoir to define the $B_{\text {loc }}\left(R^{m}\right)$ space. Since the notion of derivation is not defined on locally compact groups, we cannot use the direct generalization of the space of tempered distributions $S\left(R^{m}\right)$. On the other hand the concept of Schwartz-Bruhat spaces (cf. [1]) of rapidly decreasing smooth functions imposes too stringent conditions on test functions, while their topological dual spaces of tempered distributions, involving Frechet-type spaces, are too general for use in the real situations. Therefore, we follow the wellknown Feichtinger algebra track (cf. [6]) to develop a Banach space $\mathscr{H}_{w}^{p}(\mathscr{G})$ 
of test functions and the space of its antifunctionals $\mathscr{H}_{w}^{p^{\sim}}(\mathscr{G})$, with $\mathscr{G}$ being a locally compact group and $1 \leq p<\infty$, on the lines of Feichtinger and Gröchenig [15]. Moreover, by virtue of the inclusion relations

$$
\left.\mathscr{H}_{w}^{p} \subseteq \mathscr{H}_{w}^{1} \subseteq\left(\mathscr{H}_{w}^{1}\right)\right\urcorner \subseteq \mathscr{H}_{w}^{p^{\sim}}(\mathscr{G}),
$$

our reservoir spaces $\mathscr{H}_{w}^{p^{\sim}}(\mathscr{G})$ are larger than that of Feichtinger and Gröchenig [15]. We define the Wiener amalgam $W(B, Y)(G)$ on the lines of Feichtinger using the space $\mathscr{H}_{w}^{p^{\sim}}(\mathscr{G})$ in place of $S\left(R^{m}\right)$.

The problem of compactness in $L^{p}$-spaces on locally compact groups was studied by Weil [20]. The corresponding results for Orlicz spaces have been discussed by Bund [2]. More general results on compactness in Köthe spaces have been obtained by Goes and Welland [17]. The compactness problems for various spaces of differentiable functions on the Euclidean spaces have been studied by Nikol'skiǐ [18].

Feichtinger [9] has studied in detail the problem of compactness in translation-invariant Banach spaces of distributions on locally compact groups. His theorems are more general than earlier results in this line of work and hold true even for the space of ultra distributions on locally compact groups. He has pointed out that compactness criteria hold for a family of much more general Banach spaces of distributions defined by decomposition methods, including the usual Besov spaces (cf. [13]). Feichtinger has also discussed in [13] the applications of his results for the study of compact multipliers on translation-invariant Banach spaces of functions or distributions on locally compact groups.

The purpose of this paper is to study the compactness of bounded subsets of a general Wiener amalgam $W(B, Y)(\mathscr{G})$, where the local and global components $B$ and $Y$ are both solid Banach function (BF) spaces and satisfy some other suitable conditions. In Section 2, we give the necessary definitions and concepts for use in Section 3 which deals with the weighted Banach spaces, and in Section 4 , we describe the partition of unity on $\mathscr{G}$. In Section 5 , we define the Wiener amalgams $W(B, Y)(\varphi)$ and in Proposition 5.2, we mention some useful properties of these spaces. In Section 6, we define spline quasi-interpolation operators and state two useful lemmas. Section 7 is devoted to the study of tightness and equicontinuity of a bounded subset in a Wiener amalgam space. Theorem 8.1 is the main result of this paper, which provide a generalization of the corresponding results of Feichtinger (cf. [9, Theorem 2.2] and [12, Proposition 5, page 131]).

2. Preliminaries. We suppose that $\mathscr{G}$ is a locally compact group and $\Gamma$ its dual group consisting of all continuous characters on $\mathscr{G}$. We denote by $d x$ and $d \gamma$ the normalized Haar measures on $\mathscr{G}$ and $\Gamma$, respectively. We also assume that $\mathscr{G}$ is a $\sigma$-compact group so that all sums, coverings, and index sets on $\mathscr{G}$ 
are countable. We define the left- and right- translation operators on $\mathscr{G}$ by

$$
\begin{aligned}
& L_{x} f(y)=f(y-x) \quad \forall x, y \in \mathscr{G}, \\
& R_{x} f(y)=f\left(x y^{-1}\right) \Delta^{-1}(y),
\end{aligned}
$$

and the involution functions $\breve{f}$ and $f^{\nabla}$ by

$$
\breve{f}(x)=f(-x), \quad f^{\nabla}(x)=\overline{f(-x)},
$$

where $\nabla$ denotes the Haar modular function on $\varphi$.

We denote the Fourier transform of $f$ on $\mathscr{G}$ by $\hat{f}$ such that

$$
\hat{f}(\gamma)=\int_{\mathscr{G}}(-x, \gamma) f(x) d x .
$$

We denote by $C^{0}(\mathscr{G})$ the space of all continuous functions on $\mathscr{G}$ vanishing at infinity and by $C_{C}(\mathscr{G})$ the topological vector space of all continuous functions on $\mathscr{G}$ with compact support. The space $C_{C}(\mathscr{G})$ is endowed with its natural inductive limit topology and its topological dual can be identified with $R(\mathscr{G})$, the space of all Radon measures on $\mathscr{G}$. Also, the closure of $C_{\mathcal{C}}(\mathscr{G})$ in $L^{\infty}(\mathscr{G})$ is identified with $C^{0}(\mathscr{G})$. The space $L_{\text {loc }}^{1}(\mathscr{G})$ of all locally integrable functions on $\mathscr{G}$ is considered as a closed subspace of $R(\mathscr{G})$, that is, two measurable functions which coincide locally almost everywhere (l.a.e) are identical as usual. This implies that the topology on $L_{\text {loc }}^{1}(\mathscr{G})$ is generated by the system of seminorms $\left\{s_{K}\right\}$ given by

$$
s_{K}(f)=\int_{K}|f(x)| d x
$$

where $K$ varies over the family of all compact subsets of $\mathscr{G}$.

3. Weighted Banach spaces on $\mathscr{G}$. We assume that $m: \mathscr{G} \rightarrow \mathbb{R}_{+}$is a submultiplicative weight function on $\mathscr{G}$. It is well known that every submultiplicative weight function is locally bounded.

A locally integrable function $w: \varphi \rightarrow \mathbb{R}_{+}$is called a right moderate weight function provided that there exists a submultiplicative weight function $m$ on $\varphi$ such that

$$
w(x+y) \leq w(x) m(y) \quad \forall x, y \in \mathscr{G}
$$

We also assume that all weight functions are symmetric and satisfy the nonquasianalyticity Beurling-Domer condition (BD condition) (cf. [19, Chapter VI, Section 3]). It can easily be seen that every moderate weight function is locally bounded. 
We denote by $L_{w}^{p}(\mathscr{G}), 1 \leq p<\infty$, the Banach space of functions on $\mathscr{G}$ with respect to the norm

$$
\|f\|_{p, w} \equiv\left\|f \mid L_{w}^{p}\right\|=\left(\int_{\mathscr{G}}|f(x)|^{p} w^{p}(x) d x\right)^{1 / p}<\infty .
$$

In case $p=\infty$, we denote by $L_{w}^{\infty}(\mathscr{G})$ the Banach space of all measurable functions $f$ on $\mathscr{G}$ under the norm

$$
\left\|f \mid L_{w}^{\infty}\right\|=\operatorname{ess} \sup \{|f(x)| w(x): x \in \mathscr{G}\}<\infty
$$

The conjugate space of $L_{w}^{p}(\mathscr{G})$ is the space $L_{w^{\prime}}^{p^{\prime}}(\mathscr{G})$, where $w^{\prime}=w^{p /(1-p)}$ and $1 / p+1 / p^{\prime}=1$. It can easily be seen that $L_{w}^{p}(\varphi), 1<p<\infty$, is a reflexive Banach space and $L_{w}^{1}(\mathscr{G})$ is a Banach algebra under convolution, which is known as Beurling algebra,

$$
\begin{gathered}
L_{w}^{p} * L_{w}^{1} \subseteq L_{w}^{p}, \\
\left\|( g * f ) | L _ { w } ^ { p } \| \leq \| g | L _ { w } ^ { 1 } \left|\left\||| f \mid L_{w}^{p}\right\|\right.\right.
\end{gathered}
$$

for all $g \in L_{w}^{1}(\varphi)$ and $f \in L_{w}^{p}(\varphi)$.

Also, it can be verified that the space $L_{w}^{p}(\mathscr{G})$ is invariant under left- and righttranslation operators $L_{x}$ and $R_{x}$, respectively, and the operator norms satisfy the condition

$$
m(x) \geq\left\{\left\|\left|L_{x}\right| L_{w}^{p}|||,|\left|R_{x}\right| L_{w}^{p} \mid\right\|\right\}
$$

Let $\left(B,\|\cdot\|_{B}\right)$ be a Banach space of measurable function on $\mathscr{G}$. The space $B$ is called a BF space provided that it is continuously embedded into $L_{\text {loc }}^{1}(\mathscr{G})$. The space $B$ is known as a solid BF space if it satisfies the following solidity condition: $f \in B, g \in L_{\text {loc }}^{1}(\mathscr{G})$ with $|g(x)| \leq|f(x)|$ l.a.e. $\Rightarrow g \in B,\|g\|_{B} \leq\|f\|_{B}$.

The $L^{p}$-spaces are simple examples of solid BF spaces.

We write

$$
\begin{gathered}
u(x)=\left\|\left|L_{x}\right| B \mid\right\|, \\
v(x)=\Delta\left(x^{-1}\right)\left\|\left|R_{x^{-1}}\right| B \mid\right\|, \\
w(x) \geq c \max \left\{u(x), u\left(x^{-1}\right), v(x), v\left(x^{-1}\right) \Delta\left(x^{-1}\right)\right\}, \quad 1 \leq w(x)<\infty, \\
w(x)=w\left(x^{-1}\right) \Delta\left(x^{-1}\right) .
\end{gathered}
$$

By virtue of the closed graph theorem, the operators $L_{x}$ and $R_{x}$ are bounded on $B$ for each $x$ in $\mathscr{G}$. Thus the maps $w: x \rightarrow\left\|\left|L_{x}\right| B \mid\right\|$ and $w: x \rightarrow\left\|\left|R_{x}\right| B \mid\right\|$ are well defined and submultiplicative on $\mathscr{G}$.

4. Partition of unity on $\mathscr{G}$. It is well known that the partition of unity provides a very useful tool for discrete descriptions on a locally compact group. 
We suppose that $U$ is a compact neighbourhood of the identity $e$ in $\mathscr{G}$. A family $X=\left(x_{i}\right)_{i \in I} \in \mathscr{G}$ is called $U$-dense provided that $\left(x_{i} U\right)_{i \in I}$ covers $\mathscr{G}$, that is, $\cup_{i \in I} x_{i} U=\mathscr{G}$.

The family $X$ is called separated if the sets $\left(x_{i} U\right)_{i \in I}$ are pairwise disjoint, that is, $x_{i} U \cap x_{j} U=\phi$ for $i \neq j$.

The family $X$ is called relatively separated provided that it is a finite union of separated sets.

A family $\Psi=\left(\psi_{i}\right)_{i \in I}$ in $C^{0}(\mathscr{G})$ is called a bounded uniform partition of unity subordinate to $U$ or of size $U(U$-BUPU) provided that the following conditions hold:

(i) $X=\left(x_{i}\right)_{i \in I}$ is a relatively separated family in $\varphi$;

(ii) $0 \leq \psi_{i}(x) \leq 1$ for all $i \in I, x \in \mathscr{G}$;

(iii) $\operatorname{supp} \psi_{i} \in x_{i} U$ for all $i \in I$;

(iv) $\sum_{i \in I} \psi_{i}(x)=1$.

As a consequence of condition (iv), we have

$$
\cup_{i \in I} x_{i} U=\varphi
$$

which implies that the family $X=\left(x_{i}\right)_{i \in I}$ is $U$-dense.

5. Wiener amalgams on $\mathscr{G}$. Let $(\pi, \mathscr{H})$ be an irreducible continuous unitary representation of a locally compact group $\mathscr{G}$ on a Hilbert space $\mathscr{H}$. If $f, g \in \mathscr{H}$, the wavelet transform of $f$ with respect to $g$ is given by

$$
V_{g} f: x \rightarrow\langle\pi(x) g, f\rangle .
$$

The representation $\pi$ is called integrable provided that $V_{g} g \in L^{1}(\mathscr{G})$ for all $g \in \mathscr{H}$.

On the lines of Feichtinger and Gröchenig [15], we define the set of analyzing vectors $h_{w}^{p}(\mathscr{G})$ by

$$
h_{w}^{p}(\mathscr{G})=\left\{g: g \in \mathscr{H}, V_{g} g \in L_{w}^{p}(\mathscr{G})\right\}, \quad 1 \leq p<\infty .
$$

Now, for a fixed $g \in h_{w}^{p}(\varphi)$, we define

$$
\mathscr{H}_{w}^{p}(\mathscr{G})=\left\{f: f \in \mathscr{H}_{,}, V_{g} f \in L_{w}^{p}(\mathscr{G})\right\}
$$

and equip it with the norm

$$
\left\|f\left|\mathscr{H}_{w}^{p}\|=\| V_{g} f\right| L_{w}^{p}\right\|, \quad 1 \leq p<\infty .
$$

Following Feichtinger and Gröchenig (cf. [14, Lemma 4.2]), it can be verified that $\mathscr{H}_{w}^{p}(\mathscr{G})$ is a Banach space of distributions which are dense in $\mathscr{H}$, and the embeddings

$$
\mathscr{H}_{w}^{p}(\mathscr{G}) \hookrightarrow \mathscr{H}_{\hookrightarrow} \mathscr{H}_{w}^{p \sim}(\mathscr{G})
$$


are continuous, where $\mathscr{H}_{w}^{p}(\mathscr{G})$ is the space of all continuous conjugate-linear functionals on $\mathscr{H}_{w}^{p}(\mathscr{G})$. Also, the definitions of $\mathscr{H}_{w}^{p}(\mathscr{G})$ and $\mathscr{H}^{p}{ }_{w}^{\sim}(\mathscr{G})$ are independent of the choice of $g$ in $h_{w}^{p}(\mathscr{G})$. Since the inner product on $\mathscr{H} \times \mathscr{H}$ extends to a sesquilinear form on $\mathscr{H}_{w}^{p} \times \mathscr{H}_{w}^{p}{ }^{\sim}$, the extended wavelet transforms

$$
V_{g} f(x)=\langle\pi(x) g, f\rangle
$$

are well defined for all $g \in \mathscr{H}_{w}^{p}(\varphi)$ and $\mathscr{H}_{w}^{p \sim}(\mathscr{\varphi})$.

Now, on the lines of Feichtinger [12, page 124], we introduce the concept of uniformly localizable Banach space on $\mathscr{G}$.

DEFINITION 5.1. A Banach space $\left(B,\|\cdot\|_{B}\right)$ on $\mathscr{G}$ is said to be uniformly localizable if the following conditions hold:

(i) the embeddings $\mathscr{H}_{w}^{p}(\mathscr{G}) \hookrightarrow B \hookrightarrow \mathscr{H}_{w}^{p} \sim(\mathscr{G})$ are continuous;

(ii) for every $g \in \mathscr{H}_{w}^{p}(\mathscr{G})$,

(a) $g \cdot f \in B$ for all $f \in B$,

(b) $\left\|L_{x} g \cdot f\right\|_{B} \leq C\|f\|_{B}$ for all $x \in \mathscr{G}$ and $f \in B$, where $C$ is a positive constant depending on $g$.

The Banach space $\left(B,\|\cdot\|_{B}\right)$ is also assumed to be isometrically left translation invariant and $L_{w}^{1}(\mathscr{G})$-module in the algebraic sense, that is,

$$
\begin{gathered}
\left\|L_{x} f\right\|_{B}=\|f\|_{B} \quad \forall f \in B, \\
L_{w}^{1} * B \subseteq B, \\
\|g * f\|_{B} \leq\left\|g \mid L_{w}^{1}\right\|\|f\|_{B} .
\end{gathered}
$$

The space $\left(B,\|\cdot\|_{B}\right)$ is called a local component.

Next, a global component $\left(Y,\|\cdot\|_{Y}\right)$ on $\mathscr{G}$ is defined such that

(i) $Y$ is a solid BF space;

(ii) $Y$ is left and right translation invariant in the sense of

$$
\left\|L_{x} f\right\|_{Y} \leq C \cdot w\|f\|_{Y}
$$

and a similar inequality for the right translation;

(iii) $Y$ is a Banach convolution module with respect to the Beurling algebra $L_{w}^{1}(\varphi)$.

Using $B$ and $Y$ as above,

$$
W(B, Y)(\mathscr{G})=\left\{f: f \in B_{\mathrm{loc}}(\mathscr{G}), K(\phi, f) \in Y\right\}
$$

is said to be a Wiener amalgam space, where

$$
B_{\mathrm{loc}}(\mathscr{G})=\left\{f: f \in \mathscr{H}_{w}^{p}(\mathscr{G}), \phi f \in B, \forall \phi \in \mathscr{H}_{w}^{p}(\mathscr{G})\right\}
$$

and the control function $K(\phi, f)$ is given by

$$
K(\phi, f)(x)=\left\|L_{x} \phi \cdot f\right\|_{B} .
$$


The space $W(B, Y)(\mathscr{G})$ is endowed with the norm

$$
\|f \mid W(B, Y)\|=\|K(\phi, f)\|_{Y} .
$$

Since $B$ and $Y$ are solid Banach spaces, it can easily be seen that the space $W(B, Y)$ is solid.

The following proposition gives some useful properties of Wiener amalgam spaces.

Proposition 5.2. (i) The space $W(B, Y)(G)$ is continuously embedded in $B_{\text {loc }}(\mathscr{G})$.

(ii) The space $W(B, Y)(G)$ is a Banach space under the norm (5.12).

(iii) The space $W(B, Y)(G)$ is a Banach convolution module with respect to the Beurling algebra $L_{w}^{1}(\mathscr{G})$.

The proof follows on the lines of Feichtinger [7] (see also [3, Section 5]).

6. Spline quasi-interpolation operators. If $X=\left(x_{i}\right)_{i \in I}$ is a countable discrete set of sampling points in $\mathscr{G}$ and $U$ is a compact neighbourhood of the identity $e$ in $\mathscr{G}$, then the $U$-oscillation of a function $f \in B$ is defined by

$$
f_{U}^{\#}(x)=\sup _{x \in U}|f(x+u)-f(x)|,
$$

where $f^{\#}(x)$ denotes the local maximal of a function $f$ such that

$$
f^{\#}(x)=\sup _{x \in U}|f(x)|
$$

If $f \in B$, then it is obvious that $f^{\#}$ and $f_{U}^{\#}$ both belong to $B$ and we have

$$
\begin{aligned}
& (f * g)^{\#} \leq|f| * g^{\#}(x), \\
& (f * g)_{U}^{\#} \leq|f| * g_{U}^{\#}(x) .
\end{aligned}
$$

Now, on the lines of Feichtinger [12, page 127], we define a spline quasiinterpolant for any continuous function $f$ on $\mathscr{G}$ with respect to a $U$-BUPU family $\Psi=\left(\psi_{i}(x)\right)_{i \in I}$ in the form

$$
\mathrm{Sp}_{\Psi} f=\sum_{i \in I} f\left(x_{i}\right) \psi_{i}(x),
$$

which may be regarded as an irregular spline approximation of $f$. Since

$$
\left|\mathrm{Sp}_{\Psi} f\right| \leq f^{\#}
$$

we have

$$
\left\|\mathrm{Sp}_{\Psi} f\left|B\|\leq\| f^{\#}\right| B\right\|
$$


We also define a discretization operator mapping locally integrable functions into discrete measures by

$$
D_{\Psi} f=\sum_{i \in I}\left\langle f, \psi_{i}\right\rangle \delta_{x_{i}} \quad \forall f \in L_{\mathrm{loc}}^{1}(\mathscr{G})
$$

We give the following two results on the properties of spline quasi-interpolant corresponding to those of Feichtinger [12] on any $n$-dimensional Euclidean space.

LEMMA 6.1. If $C^{0}(\varphi)$ is the space of all continuous functions vanishing at infinity, then

$$
\left\|\mathrm{Sp}_{\Psi} f\left|W\left(C^{0}, Y\right)\left\|\leq C_{U}\right\| f\right| W\left(C^{0}, Y\right)\right\|
$$

with $C_{U}$ being a constant depending on $U$.

LEMMA 6.2. If $f \in W\left(C^{0}, Y\right)(\mathscr{G})$, then $f_{U}^{\#} \in W\left(C^{0}, Y\right)$ and

$$
\left\|\left(\mathrm{Sp}_{\Psi} f-f\right) \mid W\left(C^{0}, Y\right)\right\| \longrightarrow 0 \quad \text { as } U \longrightarrow e,
$$

provided that $C_{C}(\mathscr{G})$ is a dense subspace of $Y$.

The proof of these lemmas follows on the lines of Feichtinger [12].

7. Tightness and equicontinuity in Wiener amalgam spaces on $\mathscr{G}$. On the lines of Feichtinger (cf. [12, page 129]), we say that a bounded set $M$ in a Banach space $\left(B,\|\cdot\|_{B}\right)$ is (uniformly) tight with respect to $\mathscr{H}_{w}^{p}(\mathscr{G})$ provided that, for any given $\epsilon>0$, there exists $h \in \mathscr{H}_{w}^{p}(\mathscr{G})$ satisfying the following conditions:

(a) $\|h \cdot f|B\|\leq C\| f| B\|$ for all $f \in B$;

(b) $\|h \cdot f-f|B\|\leq \epsilon\| f| B\|$ for all $f \in M$.

A bounded subset $M$ in $B$ is called equicontinuous if, for any given $\epsilon>0$, there exists a compact neighbourhood $U$ of the identity $e$ in $\varphi$ such that

$$
\left\|L_{x} f-f \mid B\right\| \leq \epsilon \quad \forall f \in M, x \in U
$$

Now, in order to characterize equicontinuity in the Wiener amalgam space $W\left(C^{0}, Y\right)$, we prove a result which corresponds to that of Feichtinger [12, page 129] over Euclidean spaces.

Precisely, we prove the following theorem.

THEOREM 7.1. If $M$ is a bounded subset of $W\left(C^{0}, Y\right)(\varphi)$, then the following statements are equivalent:

(i) $M$ is left equicontinuous in $W\left(C^{0}, Y\right)$;

(ii) for any given $\epsilon>0$, there exists $\phi \in \mathscr{H}_{w}^{p}(\varphi)$ such that

$$
\left\|\phi * f-f \mid W\left(C^{0}, Y\right)\right\| \leq \epsilon \quad \forall f \in M ;
$$


(iii) for every $\epsilon>0$, there exists a neighbourhood $U_{0}$ of e such that

$$
\left\|f_{U}^{\#} \mid Y\right\| \leq \epsilon \quad \forall f \in M, U \subseteq U_{0}
$$

(iv) for any given $\epsilon>0$, there exists a neighbourhood $U_{1}$ of e such that

$$
\left\|f_{U}^{\#} \mid W\left(C^{0}, Y\right)\right\| \leq \epsilon \quad \forall f \in M, U \subseteq U_{1} ;
$$

(v) the family of spline quasi-interpolants $\left\{\mathrm{Sp}_{\Psi} f\right\}$ is uniformly convergent to $f$ in the norm topology of $W\left(C^{0}, Y\right)$.

Proof. (i) $\Rightarrow$ (ii). Let $M$ be an equicontinuous subset of $W\left(C^{0}, Y\right)(\varphi)$. We choose a neighbourhood $U$ of the identity $e$ in $\mathscr{G}$ such that

$$
\left\|L_{x} f-f \mid W\left(C^{0}, Y\right)\right\| \leq \epsilon \quad \forall f \in M, x \in U .
$$

We assume that $\phi$ is a nonnegative function in $C_{\mathcal{C}}(\mathscr{G})$ such that

$$
\int_{\mathscr{G}} \phi(x) d x=1, \quad \operatorname{supp} \phi \subseteq U \Longrightarrow \phi \in \mathscr{H}_{w}^{p}(\mathscr{G}) .
$$

Then we have

$$
\begin{aligned}
& \left\|\left(\phi * f-f \cdot \int_{\mathscr{G}} \phi(y) d y\right)\left|W\left(C^{0}, Y\right)\left\|\leq \int_{\mathscr{G}}\right\| L_{y} f-f\right| W\left(C^{0}, Y\right)\right\| \phi(y) d y \\
& \Longrightarrow\left\|\phi * f-f \mid W\left(C^{0}, Y\right)\right\| \leq \epsilon \quad \forall f \in M(\text { by }(7.5)) .
\end{aligned}
$$

(ii) $\Rightarrow$ (iii). We assume that (ii) holds. Then, by (6.4), we obtain

$$
(\phi * f)_{U}^{\#} \leq \phi_{U}^{\#} *|f|,
$$

which implies that

$$
\begin{aligned}
& f_{U}^{\#}(x) \leq(\phi * f)_{U}^{\#}(x)+(f-f * \phi)_{U}^{\#}(x) \\
& \Rightarrow \quad\left\|f_{U}^{\#}\left|Y\|\leq\|(\phi * f)_{U}^{\#}\right| Y\right\|+\left\|(f-f * \phi)_{U}^{\#} \mid Y\right\| \\
& \quad \leq\left\|\phi_{U}^{\#}\left|L_{w}^{1}\|\| f\right| Y\right\|+\frac{\epsilon}{2} \\
& \quad<\frac{\epsilon}{2}+\frac{\epsilon}{2} \quad \forall f \in m, U \subseteq U_{0} .
\end{aligned}
$$

(iii) $\Rightarrow$ (iv). We have

$$
\left\|f_{U}^{\#}\left|W\left(C^{0}, Y\right)\|\leq\|(\phi * f)_{U}^{\#} W\left(C^{0}, Y\right)\|+\|(f-\phi * f)_{U}^{\#}\right| W\left(C^{0}, Y\right)\right\| .
$$

But we see that

$$
\begin{aligned}
\left\|(\phi * f)_{U}^{\#} \mid W\left(C^{0}, Y\right)\right\| & \leq \sup _{u \in U}\left|\phi_{U}^{\#}(u)\right| \cdot\left\|f \mid W\left(C^{0}, Y\right)\right\| \\
& <\frac{\epsilon}{2} \text { by }(6.4) .
\end{aligned}
$$


Also, we have

$$
\left\|(f-\phi * f)\left|W\left(C^{0}, Y\right)\|\leq 2\| K(1-\phi, f)\right| W\left(C^{0}, Y\right)\right\|<\frac{\epsilon}{2} .
$$

Thus we infer that (7.4) holds.

(iv) $\Rightarrow$ (v). By (6.1) and Lemma 6.2, we have $\left|\mathrm{Sp}_{\Psi} f-f\right| \leq f_{U}^{\#}$ for any $U$-BUPU family $\Psi$, which implies that

$$
\left|\sum_{i \in I}\left(f\left(x_{i}\right) \psi_{i}(x)-f(x) \psi_{i}(x)\right)\right| \leq \sum_{i \in I} f_{U}^{\#} \cdot \psi_{i}(x) \quad \forall x \in \mathscr{G},
$$

that is,

$$
\left\|\mathrm{Sp}_{\Psi} f-f \mid W\left(C^{0}, Y\right)\right\| \longrightarrow 0 \quad \text { as } U \longrightarrow e .
$$

(v) $\Rightarrow$ (i). We assume that $\Psi$ is $U$-BUPU such that $\left(x_{i} U\right)_{i \in I}$ is a covering of $\varphi$ and the functions $\left(\psi_{i}\right)_{i \in I}$ form an equicontinuous subfamily in $C^{0}(\mathscr{G})$.

Now, we choose a nonnegative function $\phi$ such that

$$
\operatorname{supp} \phi \subseteq U_{3}, \quad \phi(t)=1 \text { on } U_{2},
$$

where $U_{1} \subset U_{2} \subset U_{3}\left(U_{i}, i=1,2,3\right)$ are compact neighbourhoods of the identity $e \in \varphi$.

Thus we see that

$$
\psi_{i}(x-y)=\psi_{i}(x-y) L_{x_{i}} \phi(x) \text { for } \operatorname{supp}\left(L_{y} \psi_{i}\right) \subseteq U_{2} .
$$

Hence we obtain

$$
\begin{aligned}
\left(\operatorname{Sp}_{\Psi} f\right)_{U}^{\#}(x) & =\sup _{y \in U}\left|\operatorname{Sp}_{\Psi} f(x-y)-\operatorname{Sp}_{\Psi} f(x)\right| \\
& \leq \sum_{i \in I}\left|f\left(x_{i}\right)\right| \sup _{y \in U}\left|\psi_{i}(x-y)-\psi_{i}(x)\right| \cdot\left|L_{x_{i}} \phi(x)\right| \\
& \leq \sum_{i \in I}\left|f\left(x_{i}\right)\right|\left\|\left(\psi_{i}\right)_{U}^{\#}\right\|_{\infty} \cdot L_{x_{i}} \phi(x) .
\end{aligned}
$$

Since the family $\left(\psi_{i}\right)_{i \in I}$ is equicontinuous, for any given $\eta>0$, there exists a neighbourhood $U$ of the identity $e \in \mathscr{G}$ such that

$$
\left\|\left(\psi_{i}\right)_{U}^{\#}\right\|_{\infty} \leq \eta
$$

Next, since the discrete measure $\sum_{i \in I} \delta_{x_{i}}$ belongs to $W\left(M, L^{\infty}\right)(\mathscr{G})$, we have

$$
\begin{aligned}
& \left\|\left(\operatorname{Sp}_{\Psi} f\right)_{U}^{\#} \mid W\left(C^{0}, Y\right)\right\| \\
& \quad \leq \sup _{i \in I}\left\|\left(\psi_{i}\right)_{U}^{\#}\right\|_{\infty} \cdot\left\|\left|f\left(x_{i}\right)\right| \delta_{x_{i}} * \phi \mid W\left(C^{0}, Y\right)\right\| \\
& \quad \leq \eta \cdot C\left\|\sum_{i \in I} \delta_{x_{i}}\left|W\left(M, L^{\infty}\right)\|\cdot\| f\right| W\left(C^{0}, Y\right)\right\| \cdot\left\|\phi \mid W\left(C^{0}, L^{1}\right)\right\| .
\end{aligned}
$$


Finally, since $M$ is bounded in $W\left(C^{0}, Y\right)$, we may choose $\eta$ such that

$$
\left\|\left(\mathrm{Sp}_{\Psi} f\right)_{U}^{\#}\left|W\left(C^{0}, Y\right)\|\leq \epsilon \Longrightarrow\| f_{U}^{\#}\right| W\left(C^{0}, Y\right)\right\| \leq \epsilon \quad \forall f \in M
$$

which implies that $M$ is equicontinuous in $W\left(C^{0}, Y\right)$.

This completes the proof of the theorem.

8. Compactness in Wiener amalgams. Feichtinger [12, page 131] has obtained some suitable conditions which ensure the compactness of $M \subseteq W(B$, $Y$ ) when $B$ and $Y$ are defined over $m$-dimensional Euclidean spaces. In this section, we study the compactness of a subset of the Wiener amalgam space $W(B, Y)(G)$ defined on a locally compact group $\mathscr{G}$. Our theorem provides a generalization of the corresponding result of Feichtinger [12, page 131].

Precisely, we prove the following theorem.

THEOREM 8.1. If $\mathscr{H}_{w}^{p}(\mathscr{G})$ is dense in both $B$ and $Y$, then a closed bounded set $M \subseteq W(B, Y)(\varphi)$ is compact if and only if it is uniformly tight and equicontinuous under the $W(B, Y)$-norm.

We will use the following lemma in the proof of our theorem.

LEMMA 8.2. If $C_{C}(G)$ is dense in $Y$ and the map $y \rightarrow L_{y} f$ is continuous in $B$ for all $f \in B$, then $y \rightarrow L_{y} f$ is continuous in $W(B, Y)(G)$ for all $f \in W(B, Y)$.

PROOF. The proof follows on the lines of Feichtinger [7, pages 514-515]. As our parameters are different, it is necessary to give a proof.

Since $C_{C}(\mathscr{G})$ is dense in $Y$, there exists a compact set $K_{0} \in \mathscr{G}$ such that

$$
\left\|\left(1-\chi_{K_{0}}\right) \cdot K(\phi, f) \mid Y\right\| \leq \epsilon
$$

where $\chi_{K_{0}}$ is the characteristic function of $K_{0}$.

Next, since the left translation $L_{y}$ is continuous, and so locally bounded, and since $U$ is relatively compact, we have

$$
\left\|\left|L_{y}\right|\right\|_{B} \cdot\left\|\left|L_{y}\right|\right\|_{Y} \leq C \quad \forall y \in U
$$

with $C$ being a positive constant. Thus, for all $y \in U$, we have

$$
\begin{aligned}
\left\|\left(1-\chi_{U K_{0}}\right) \cdot K\left(\phi, L_{y} f\right)(z)\right\|_{Y} & =\left\|\left(1-\chi_{U K_{0}}\right)\right\|\left(L_{z} \phi\right) \cdot\left(L_{y} f\right)\left\|_{B}\right\|_{Y} \\
& \leq C \cdot\left\|\left|L_{y}\right|\right\|_{Y} \cdot\left\|\left(1-\chi_{K_{0}}\right) \cdot K(\phi, f)\right\|_{Y} \\
& <C \epsilon
\end{aligned}
$$

because $K_{0} \subseteq y^{-1} U K_{0}$ for all $y \in U$ and so $\left(1-\chi_{y^{-1} U K_{0}}\right) \leq\left(1-\chi_{K_{0}}\right)$. 
Hence we infer that, for any given $\epsilon>0$ and any open neighbourhood $U \subseteq \mathscr{G}$ with compact closure, we can find $K \subseteq \mathscr{G}$ such that

$$
\left\|\left(1-\chi_{K}\right) \cdot K\left(\phi, L_{y} f\right)\right\|_{Y} \leq \epsilon \quad \forall y \in U
$$

which implies that $\left\|\left(1-\chi_{K}\right) \cdot K(\phi, f)\right\|_{Y} \leq \epsilon$.

We now choose $h$ in $\mathscr{H}_{w}^{p}(\mathscr{G})$ such that $h=1$ on $U^{-1} K(\operatorname{supp} \phi)$. Thus we see that

$$
L_{z} \phi \cdot\left(L_{y} f-f\right)=L_{z} \phi\left[L_{y}(h f)-(h f)\right] .
$$

Next, we choose $U_{0} \subseteq U$ such that

$$
\left\|L_{y}(h f)-(h f)\right\|_{B}<\epsilon \quad \forall y \in U_{0} .
$$

Hence, on account of the above relations, we obtain

$$
\begin{aligned}
\left\|K\left(\phi, L_{y} f-f\right)\right\|_{Y} \leq & \left\|\left(1-\chi_{K}\right) \cdot K\left(\phi, L_{y} f\right)\right\|_{Y} \\
& +\left\|\left(1-\chi_{K}\right) K(\phi, f)\right\|_{Y}+\left\|\left(1-\chi_{K}\right) \cdot K\left(\phi, L_{y} f-f\right)\right\|_{Y} \\
\leq & 2 \epsilon+\left\|\chi_{K}\right\|_{Y} \cdot\left\|L_{y}(h \cdot f)-(h \cdot f)\right\|_{B} \rightarrow 0 \text { as } \epsilon \longrightarrow 0 .
\end{aligned}
$$

Hence the map $y \rightarrow L_{y} f$ is continuous from $\mathscr{G}$ into the norm topology of $W(B, Y)(\varphi)$.

Thus the lemma holds true.

Proof OF TheOrem 8.1. We suppose that $M$ is uniformly tight and equicontinuous under the $W(B, Y)$-norm. Hence, by virtue of Lemma 8.2, the left translation is continuous in $W(B, Y)(\varphi)$.

At first we will show that $M$ is relatively compact in $\mathscr{H}_{w}^{p \sim}(\mathscr{G})$. Since the embeddings

$$
\mathscr{H}_{w}^{p}(\varphi) \hookrightarrow W(B, Y)(\varphi) \hookrightarrow \mathcal{H}_{w}^{p \sim}(\mathscr{G})
$$

are continuous (the proof follows on the lines of Feichtinger [7, Theorem 1]), hence, for any compact set $K \subseteq \mathscr{G}$, there exist positive constants $C_{K}$ and $C_{K}^{\prime}$ such that

$$
\|h\|_{W(B, Y)} \leq C_{K}\|h\|_{\mathscr{H}_{w}^{p}}
$$

and, by closed graph theorem,

$$
|\langle h, f\rangle| \leq C_{K}^{\prime}\|f\|_{W(B, Y)} \cdot\left\|h \mid \mathscr{H}_{w}^{p}\right\|
$$

for all $h \in \mathscr{H}_{w}^{p}(\mathscr{G})$ with supp $h \subseteq K$ and $f \in W(B, Y)$. 
Now let $k_{0}$ be a function in $\mathscr{H}_{w}^{p}(\mathscr{G})$ such that supp $k_{0} \subseteq K$ and $\epsilon>0$ are given. Then, there exists a set $V$ such that

$$
V=\left\{k: k \in \mathscr{H}_{w}^{p}(\mathscr{G}), \operatorname{supp} k \subseteq K,\left\|k-k_{0}\right\|_{\mathscr{H}_{w}^{p}}<\epsilon\left(C_{K}^{\prime} \cdot C\right)^{-1}\right\}
$$

Thus we see that

$$
\left|\left\langle k-k_{0}, f\right\rangle\right|<\epsilon \quad \forall k \in V, \forall f \in M .
$$

This implies that $M$ is relatively compact in $\mathscr{H}_{w}^{p} \sim(\mathscr{G})$ with respect to the weak topology $\sigma\left(\mathscr{H}_{w}^{p \sim}, \mathscr{H}_{w}^{p}\right)$ (cf. [9, pages 301-302]).

Hence, for any given net in $M$, we can find a subnet $\left(f_{\alpha}\right)_{\alpha \in I}$ in $M$ and $\sigma \in$ $\mathscr{H}_{w}^{p \sim}(\mathscr{G})$ such that

$$
\lim _{\alpha \rightarrow \infty} \int_{\mathscr{G}} f_{\alpha}(x) h(x) d x=\int_{\mathscr{G}} \sigma(x) h(x) d x \quad \forall h \in \mathscr{H}_{w}^{p} \mathscr{G} .
$$

We now claim that $\left\{f_{\alpha}\right\}_{\alpha \in I}$ is a Cauchy net under the norm of $W(B, Y)(\varphi)$. Let $\epsilon>0$ be given. Then there exists $k \in \mathscr{H}_{w}^{p}(\mathscr{G})$ such that

$$
\|k * f-f\|_{W(B, Y)}<\epsilon \quad \forall f \in M .
$$

Hence we can find $h \in \mathscr{H}_{w}^{p}(\mathscr{G})$ such that

$$
\|h f-f\|_{W(B, Y)} \leq \epsilon \quad \forall f \in M .
$$

Combining (8.14) and (8.15), we get

$$
\begin{aligned}
\|k * h f-f\|_{W(B, Y)} & \leq\|k * h f-k * f\|_{W(B, Y)}+\|k * f-f\|_{W(B, Y)} \\
& <2 \varepsilon \quad \forall f \in M .
\end{aligned}
$$

Since $\mathscr{H}_{w}^{p}(\mathscr{G}) \cap W(B, Y)$ is dense in $\mathscr{H}_{w}^{p}(\mathscr{G})$, which in turn is dense in $L_{w}^{1}(\mathscr{G})$, we can find $k_{1} \in \mathscr{H}_{w}^{p}(\mathscr{G}) \cap W(B, Y)$ such that

$$
\left\|k_{1} * k-k\right\|_{1, w}<\epsilon .
$$

Thus, on account of the relation

$$
\|h f\|_{W(B, Y)} \leq\|f\|_{W(B, Y)}+\epsilon \leq 2 C,
$$

we see that

$$
\left\|f-k_{1} * k * h f\right\|_{W(B, Y)} \leq 4 \epsilon \quad \forall f \in M .
$$


Now, since $\left\{h f_{\alpha}\right\}_{\alpha \in I}$ is a convergent net of function with compact support, we can find $\alpha_{0}$ in $I$ such that

$$
\left\|k * h f_{\alpha}-k * h f_{\beta}\right\|_{1, w} \leq \frac{\epsilon}{\left\|k_{1}\right\|_{W(B, Y)}} \quad \forall \alpha, \beta \geq \alpha_{0},
$$

because $\left(k * h f_{\alpha}\right)$ is uniformly convergent on compact sets.

Thus we see that

$$
\left\|k_{1} * k * h f_{\alpha}-k_{1} * k * h f_{\beta}\right\|_{W(B, Y)}<\epsilon \quad \forall \alpha, \beta \geq \alpha_{0} .
$$

Hence, using (8.19), we obtain

$$
\left\|f_{\alpha}-f_{\beta}\right\|_{W(B, Y)} \geq C \epsilon \quad \forall \alpha, \beta \geq \alpha_{0},
$$

where $C$ is a suitably chosen positive constant.

Conversely, let $M$ be compact in $W(B, Y)(\varphi)$ and $\epsilon>0$ be given. Since $\mathscr{H}_{w}^{p}(\varphi)$ is dense in $W(B, Y)$, there exists a finite sequence $\left(f_{i}\right)_{i=1}^{n}$ in $\mathscr{H}_{w}^{p}(\mathscr{G})$ such that

$$
\left\|f-f_{i}\right\|_{W(B, Y)}<C \epsilon .
$$

Hence we can find $h$ and $k$ in $\mathscr{H}_{w}^{p}(\mathscr{G})$ such that

$$
\begin{gathered}
\|k\|_{p, w} \leq C_{w}, \\
\|h f\|_{W(B, Y)} \leq C_{w}\|f\|_{W(B, Y),} \\
\left\|h * f_{i}-f_{i}\right\|_{W(B, Y)}<\epsilon, \\
\left\|k * f_{i}-f_{i}\right\|_{W(B, Y)}<\epsilon, \quad \forall 1 \leq i \leq n .
\end{gathered}
$$

Thus we see that

$$
\begin{aligned}
\|k * f-f\|_{W(B, Y)}= & \left\|k * f-k * f_{i}\right\|_{W(B, Y)}+\left\|k * f_{i}-f_{i}\right\|_{W(B, Y)} \\
& +\left\|f_{i}-f\right\|_{W(B, Y)}=C \epsilon \quad \forall f \in M .
\end{aligned}
$$

Hence we infer that $M$ is uniformly tight and equicontinuous in the norm topology of $W(B, Y)(\mathscr{G})$. Thus the conditions are necessary.

This completes the proof of the theorem.

ACKNOWLEDGMENTS. This work was financially supported by grant no. DST/MS/150/2k. The author is thankful to the referee for various suggestions and helpful criticism which improved the presentation of the paper.

\section{REFERENCES}

[1] F. Bruhat, Distributions sur un groupe localement compact et applications à l'étude des représentations des groupes p-adiques, Bull. Soc. Math. France 89 (1961), 43-75 (French).

[2] I. M. Bund, Birnbaum-Orlicz spaces of functions on groups, Pacific J. Math. 58 (1975), no. 2, 351-359. 
[3] T. Dobler, Wienertyperaume auf local-kompakten gruppen, Diploma thesis, Institut für Mathematics der Universitat Wien, Wien, 1989.

[4] H. G. Feichtinger, A characterization of Wiener's algebra on locally compact groups, Arch. Math. (Basel) 29 (1977), no. 2, 136-140.

[5] _ Banach spaces of distributions of Wiener's type and interpolation, Functional Analysis and Approximation (Oberwolfach, 1980), Internat. Ser. Numer. Math., vol. 60, Birkhäuser, Basel, 1981, pp. 153-165.

[6] _ On a new Segal algebra, Monatsh. Math. 92 (1981), no. 4, 269-289.

[7] _ Banach convolution algebras of Wiener type, Functions, Series, Operators, Vol. I, II (Budapest, 1980), Colloq. Math. Soc. János Bolyai, vol. 35, NorthHolland, Amsterdam, 1983, pp. 509-524.

[8] _ Strong almost periodicity and Wiener type spaces, Constructive Function Theory '81 (Varna, 1981), Bulgar. Acad. Sci., Sofia, 1983, pp. 321-327.

[9]__ Compactness in translation invariant Banach spaces of distributions and compact multipliers, J. Math. Anal. Appl. 102 (1984), no. 2, 289-327.

[10]_ Generalized amalgams, with applications to Fourier transform, Canad. J. Math. 42 (1990), no. 3, 395-409.

[11] _ New results on regular and irregular sampling based on Wiener amalgams, Function Spaces (Edwardsville, Ill, 1990), Lecture Notes in Pure and Appl. Math., vol. 136, Marcel Dekker, New York, 1992, pp. 107-121.

[12] _ Wiener amalgams over Euclidean spaces and some of their applications, Function spaces (Edwardsville, Ill, 1990), Lecture Notes in Pure and Appl. Math., vol. 136, Marcel Dekker, New York, 1992, pp. 123-137.

[13] H. G. Feichtinger and P. Gröbner, Banach spaces of distributions defined by decomposition methods. I, Math. Nachr. 123 (1985), 97-120.

[14] H. G. Feichtinger and K. H. Gröchenig, A unified approach to atomic decompositions via integrable group representations, Function Spaces and Applications (Lund, 1986), Lecture Notes in Math., vol. 1302, Springer, Berlin, 1988, pp. 52-73.

[15] _ _ Banach spaces related to integrable group representations and their atomic decompositions. I, J. Funct. Anal. 86 (1989), no. 2, 307-340.

[16] J. J. F. Fournier and J. Stewart, Amalgams of $L^{p}$ and $l^{q}$, Bull. Amer. Math. Soc. (N.S.) 13 (1985), no. 1, 1-21.

[17] S. Goes and R. Welland, Compactness criteria for Köthe spaces, Math. Ann. 188 (1970), 251-269.

[18] S. M. Nikol'skiu, Compactness of classes $H_{p}^{\left(r_{1}, \cdots, r_{n}\right)}$ of functions of several variables, Izv. Akad. Nauk SSSR Ser. Mat. 20 (1956), 611-622.

[19] H. Reiter and J. D. Stegeman, Classical Harmonic Analysis and Locally Compact Groups, 2nd ed., London Mathematical Society Monographs, New Series, vol. 22, The Clarendon Press, Oxford University Press, New York, 2000.

[20] A. Weil, L'intègration dans les groupes topologiques et ses applications, Act. Sci. Ind., no. 1145, Hermann, Paris, 1951.

[21] N. Wiener, On the representation of functions by trigonometrical integrals, Math. Z. 24 (1926), 575-616.

S. S. Pandey: Department of Mathematics, Rani Durgawati University, Jabalpur, India E-mail address: shee1 pandey@hotmai 1 .com 


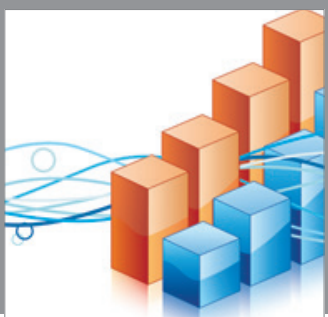

Advances in

Operations Research

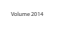

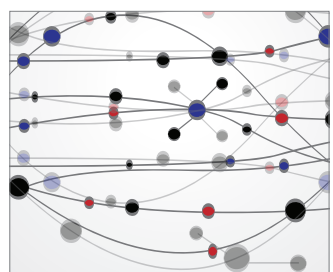

\section{The Scientific} World Journal
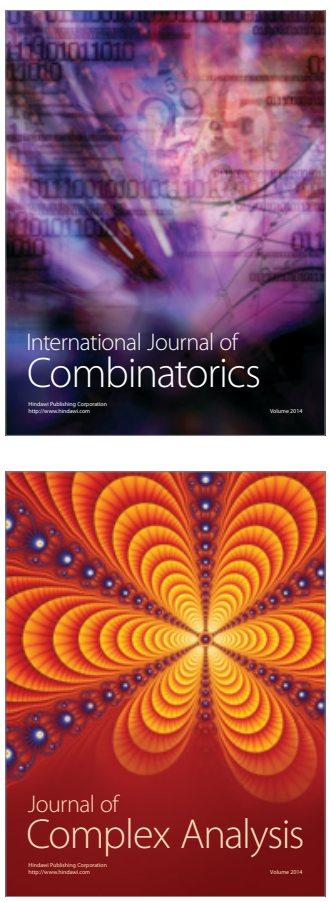

International Journal of

Mathematics and

Mathematical

Sciences
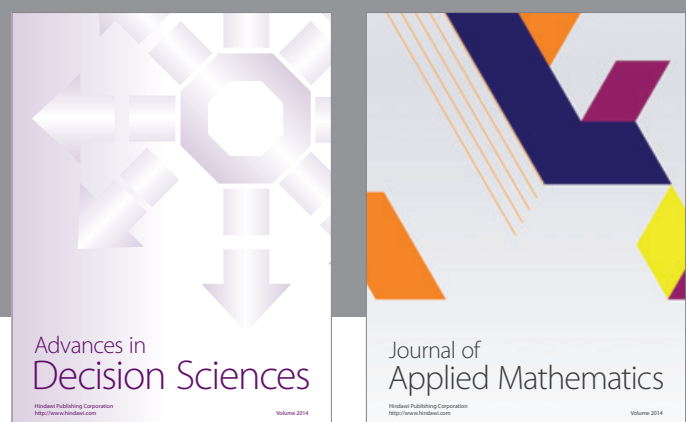

Journal of

Applied Mathematics
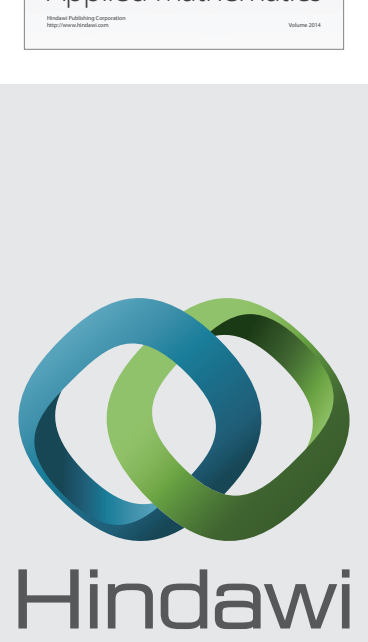

Submit your manuscripts at http://www.hindawi.com
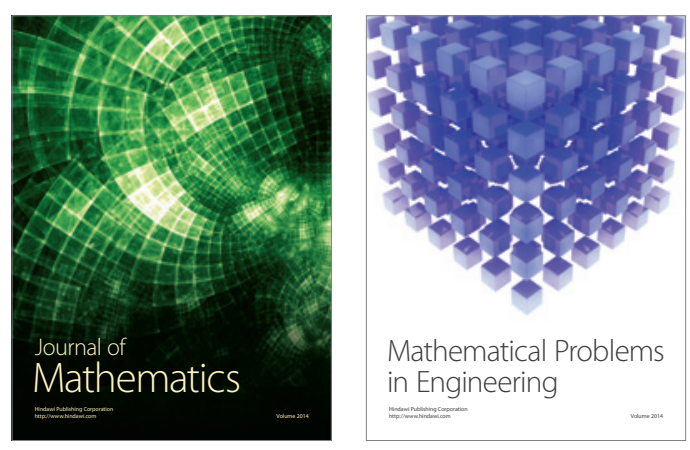

Mathematical Problems in Engineering
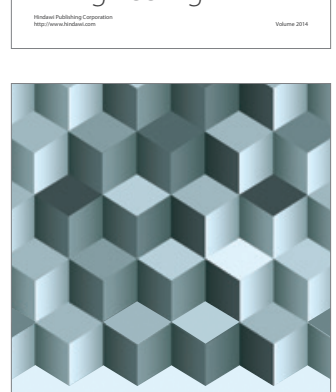

Journal of

Function Spaces
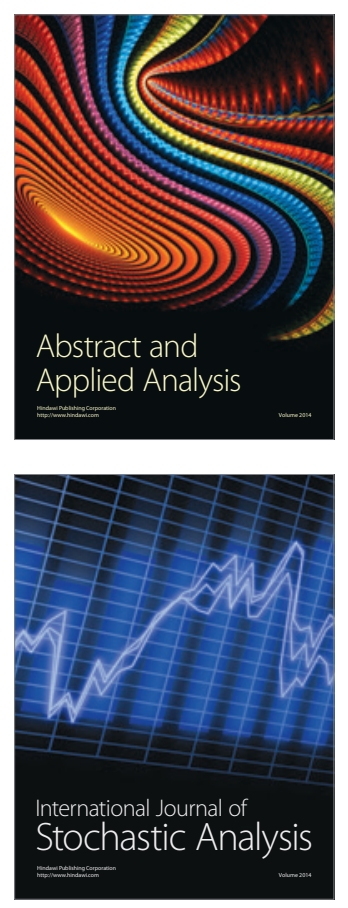

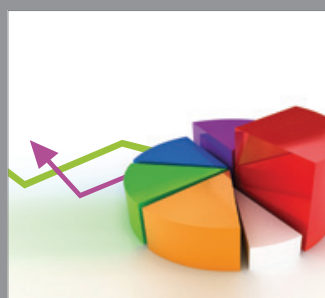

ournal of

Probability and Statistics

Promensencen
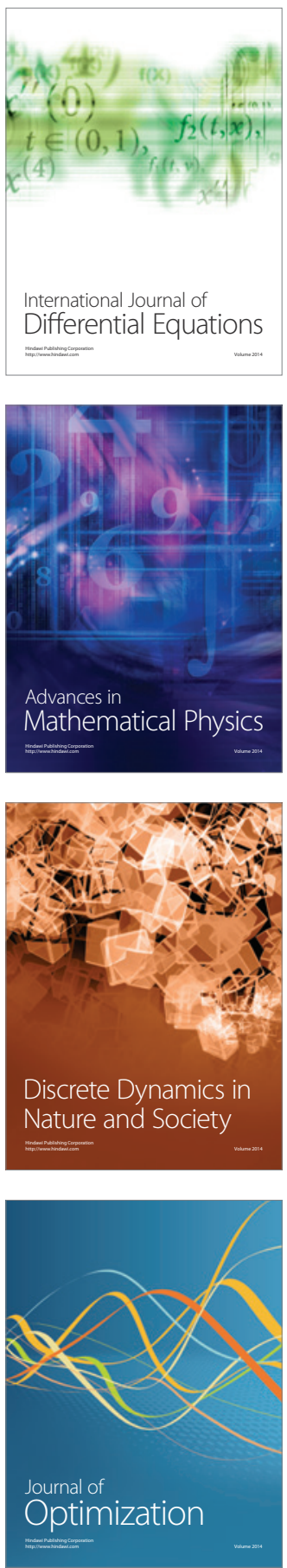